\title{
Interference of Quantum Market Strategies
}

\author{
Edward W. Piotrowski \\ Institute of Theoretical Physics, University of Białystok, \\ Lipowa 41, Pl 15424 Białystok, Poland \\ e-mail: ep@alpha.uwb.edu.pl \\ Jan Sładkowski \\ Institute of Physics, University of Silesia, \\ Uniwersytecka 4, Pl 40007 Katowice, Poland \\ e-mail: sladk@us.edu.pl \\ and Jacek Syska \\ Institute of Physics, University of Silesia, \\ Uniwersytecka 4, Pl 40007 Katowice, Poland \\ e-mail: jacek@server.phys.us.edu.pl
}

\begin{abstract}
Recent development in quantum computation and quantum information theory allows to extend the scope of game theory for the quantum world. The paper is devoted to the analysis of interference of quantum strategies in quantum market games.
\end{abstract}

PACS numbers: 02.50.Le, 03.67.-a, 03.65.Bz

Keywords: quantum games, quantum strategies, econophysics, financial markets

\section{Introduction}

Recent development in quantum computation and quantum information theory allows to extend the scope of game theory for the quantum world [1, 2]. 
In the "standard" quantum game theory one tries in some sense to quantize an operational description of the "classical" game in question. Iqbal and Toor have successfully applied the method of quantization of games proposed by Marinatto and Weber [3] in biology [4, 5, 6] and recently they have used the same formalism to analyse the Stackelberg duopoly (leader-follower model) [7]. In the classical setting of the duopoly game the follower becomes worse-off compared to the leader who becomes better-off. But they have shown that in the quantum version the follower is not hurt even if he or she knows the action of the leader. The backward induction outcome is the same as the Nash equilibrium in the classical Cournot game (that is when decisions are made simultaneously and there is no information hurting players). Two of the present authors have managed to formulate a new approach to quantum game theory that is suitable for description of market transactions in terms of supply and demand curves [8]-[11]. In this approach quantum strategies are vectors in some Hilbert space and can be interpreted as superpositions of trading decisions. For a trader they form the "quantum board". The actual subject of investigation may consist of single traders, teams of traders or even the whole market. Not the apparatus nor installation for actual playing but strategies are at the very core of the theory. Spontaneous or institutionalized market transactions are described in terms of projective operations acting on Hilbert spaces of traders' strategies. Quantum entanglement is necessary to strike the balance of trade. The discussed below text-book examples of departures from the demand and supply law are related to the negative probabilities that often emerge in quantum theories and form very interesting illustrations of them [9, 10]. This model predicts the property of undividity of the traders' attention (no clonning theorem). The theory unifies also the English auction with the Vickrey's one attenuating the motivation properties of the latter. There are analogies with quantum thermodynamics that allow to interpret market equilibrium as a state with vanishing financial risk flow. Sometimes euphoria, panic or the herd instinct cause violent changes of market prices. Such phenomena can be described by the non-commutative quantum mechanics $[8,10]$. There is a simple tactic that maximize the trader's profit on an effective market: accept profits equal or greater then the one you have formerly achieved on average. Here we would like to analyse the consequences of interference of quantum strategies. We begin with the analysis of tactics changing the demand. Then we discuss the supply-side aspects. Both sides' strategies are related by the Fourier transform. 


\section{Tactics changing demand}

Traders' strategies are represented by probability amplitudes (wave functions) $|\psi\rangle$. There are two important representations $\langle q \mid \psi\rangle$ (demand representation) and $\langle p \mid \psi\rangle$ (supply representation) where $q$ and $p$ are logarithms of prices at which the player is buying or selling, respectively $[8,11,12]$. We have defined canonically conjugate hermitian operators of demand $\mathcal{Q}$ and supply $\mathcal{P}$ corresponding to the variables $q$ and $p$ characterizing strategy of the player. This led us to the definition of the observable that we call the risk inclination operator [8]:

$$
H(\mathcal{P}, \mathcal{Q}):=\frac{\left(\mathcal{P}-p_{0}\right)^{2}}{2 m}+\frac{m \omega^{2}\left(\mathcal{Q}-q_{0}\right)^{2}}{2}
$$

where $p_{0}:=\frac{\langle\psi|\mathcal{P}| \psi\rangle}{\langle\psi \mid \psi\rangle}, q_{0}:=\frac{\langle\psi|\mathcal{Q}| \psi\rangle}{\langle\psi \mid \psi\rangle}, \omega:=\frac{2 \pi}{\theta} \cdot \theta$ is, roughly speaking, an average time spread between two opposite moves of a player (e.g. buying and selling the same commodity) [12]. The parameter $m>0$ measures the risk asymmetry between buying and selling positions. Analogies with quantum harmonic oscillator are not accidental and one can even define a parameter $h_{E}$ that describes the minimal inclination of the player to risk [8]. The distribution functions, that is integrals of squared absolute values of the amplitudes, have natural interpretation in terms of demand and supply curves [10,11]. This picture should be supplemented with dynamics resulting from the traders' tactical moves. Tactical moves of traders, that modify their behaviour on the market, are described by unitary operations on the Hilbert space of strategies $L^{2},\langle q \mid \psi\rangle \in L^{2}$. The fundamental tactic that switches the supply strategy into the demand one and vice versa is described by the Fourier transform. There are also tactics that only modify the demand or supply strategy without switching sides. We are interested in such tactics that the resulting strategy is a pure state and therefore cannot be reduced to a convex combination of strategies that is to the components having definite supply or demand characteristics. Such combinations are possible only if components are not orthogonal (that is, they do not correspond to classical strategies). The possibility of forming such combinations is a pure quantum effect that cannot be described in terms of the classical probabilistic approach.

The "linear shift" of the demand by $\Delta$

$$
\mathcal{D}_{\Delta}\langle q \mid \psi\rangle:=\langle q-\Delta \mid \psi\rangle
$$


commutes with the supply operator $\mathcal{P}[8,10]$ and therefore does not change the supply side. It only results in a phase shift of the Fourier transformed strategy, $\langle p \mid \psi\rangle \rightarrow \mathrm{e}^{\mathrm{i} \hbar_{E}^{-1} \Delta p}\langle p \mid \psi\rangle$, so the shape of the supply curve of the player is unchanged. The quantum formalism enables playing by using families of tactics of the form (the proper normalization can be achieved by respective normalization of supply and demand curves)

$$
\mathcal{D}_{\Delta}\left(\xi_{0}, \xi_{1}\right):=\xi_{0} \mathcal{I}+\xi_{1} \mathcal{D}_{\Delta}
$$

where $\left(\xi_{0}, \xi_{1}\right)$ are homogeneous complex projective coordinates of points on the Riemann sphere $S^{2} \simeq \mathbb{C} P^{1} \simeq \overline{\mathbb{C}}$, and $\mathcal{I}=\mathcal{D}_{0}$ is the identity map. The poles $(1,0)$ and $(0,1)$ correspond to identity mapping of the initial strategy and its shifted copies, respectively. The remaining points parameterize all possible superpositions of the initial strategies. We define the (commuting) composition of tactics transforming the initial strategy $\langle q \mid \psi\rangle$ as:

$$
\mathcal{D}_{\Delta^{\prime}}\left(\xi_{0}^{\prime}, \xi_{1}^{\prime}\right) \circ \mathcal{D}_{\Delta^{\prime \prime}}\left(\xi_{0}^{\prime \prime}, \xi_{1}^{\prime \prime}\right):=\mathcal{D}_{\Delta^{\prime}+\Delta^{\prime \prime}}\left(\xi_{0}^{\prime} \cdot \xi_{0}^{\prime \prime}, \xi_{1}^{\prime} \cdot \xi_{1}^{\prime \prime}\right)
$$

The transition from a state being a normal (Gaussian) distribution to a superposition of such states is not unitary but the tactics $\mathcal{D}_{\Delta}\left(\xi_{0} \neq 0, \xi_{1} \neq 0\right)$ manipulating coefficients of the superposition or the widths $\Delta$ of Gaussian components are unitary so it is convenient to use instead of the tactics $\mathcal{D}(1,0)$ and $\mathcal{D}_{\Delta}(1,0)$ series of tactics which are convergent to them.

Let us investigate the effect of the family of tactics $\mathcal{D}_{\Delta}\left(\xi_{0}, \xi_{1}\right)_{\xi_{0}, \xi_{1}}$ on the process of changing the demand (and the corresponding supply) with the effects of the interference of the strategies. They represent behaviours of the player which differ from the standard one dictated by the law of demand and supply. The diverse market strategies might be identified with the behaviours of the player connected with the price of the particular goods (which have appeared under different states of his (or her) knowledge or perhaps subconsciousness). Then the superposition of the strategies of the player could be interpreted as the appearance of the diverse types of couplings between simultaneous ensembles of different states of the player. Often during the alteration of our opinions and characteristic behaviours, the old and new stages of our changing individuality coexist with each other in a variety of relations. With the lack of such coexistence, "schizophrenic" states might lead to the behaviors which are socially inefficient. 


\section{The results of the tactic $\mathcal{D}_{\Delta}\left(\xi_{0}, \xi_{1}\right)$ for the Gaussian strategy}

We are interested in quantum description of a game which consists in buying or selling of market goods. We will investigate the pure adiabatic strategies only. Such a restriction is justified by the fact that in the market reality the inclination to the risk seems to be a characteristic feature of the player hence for one participant of the market it is unchanged even over big intervals of the time. Therefore the player has at his (her) disposal the Gaussian standard strategy, which is equal to the square root of the standard normal distribution $\langle q \mid \psi\rangle_{g}:=\frac{1}{\sqrt[4]{2 \pi}} \mathrm{e}^{-\frac{q^{2}}{4}}$ as a function of the logarithm of the price, above which the player give up buying goods offered to him (withdrawal price [10, 12]). The free choice both of the currency unit and the logarithmic base allows to choose the two first moments of the distribution $\left|\langle q \mid \psi\rangle_{g}\right|^{2}$ to be equal to $q_{0}=0$ and $\sigma=1$ respectively. It is worth to notice that the Gaussian strategy is, in the quantum setting, the only one which fulfills the law of the demand and supply.

Let the player operates with tactics which belonging to the family $\left\{\mathcal{D}_{\Delta}(z):=\right.$ $\left.\mathcal{D}_{\Delta}\left(\xi_{0}, \xi_{1}\right)\right\}$. To abbreviate the notation we use the inhomogeneous coordinate on the Riemann sphere, $\frac{\xi_{1}}{\xi_{0}}=: z \in \overline{\mathbb{C}}$. As the result of applying the tactics $\mathcal{D}_{\Delta}(z)$, the Gaussian strategy is replaced with the superposition of the Gaussian strategies, given by

$$
\mathrm{e}^{-\frac{q^{2}}{4}} \longrightarrow \mathrm{e}^{-\frac{q^{2}}{4}}+z \mathrm{e}^{-\frac{(q-\Delta)^{2}}{4}} .
$$

In the following Figures we will compare the player's demand given by the function of the probability density distribution $\left|\mathcal{D}_{\Delta}(z)\langle q \mid \psi\rangle_{g}\right|^{2}$ with the density function given $b^{1}$

$$
\frac{1}{\sqrt{2 \pi}}\left(\frac{1}{1+|z|^{2}} \mathrm{e}^{-\frac{q^{2}}{2}}+\frac{|z|^{2}}{1+|z|^{2}} \mathrm{e}^{-\frac{(q-\Delta)^{2}}{2}}\right),
$$

which would appear in the classical theory (in which the interference effects are neglected). As a result of interference, for $\arg (z)=0$, we observe the biggest overlapping of two Gaussian curves, which in addition are submitted

\footnotetext{
${ }^{1}$ In the quantum theory such a type of density functions appear in the case of the tactics $\mathcal{D}_{\Delta}(z)$, for which $z$ is imaginary $\left(\arg (z)= \pm \frac{\pi}{2}\right)$.
} 
to a mutual attraction. Figure 1 illustrates the case for $\Delta=3$ and $z=0.9$. In order to demonstrate the scale of the quantum effects, the corresponding sum of two Gaussian strategies which does not have the interference ingredient (1) is drawn in Figure 1 and the following ones as the dashed line.

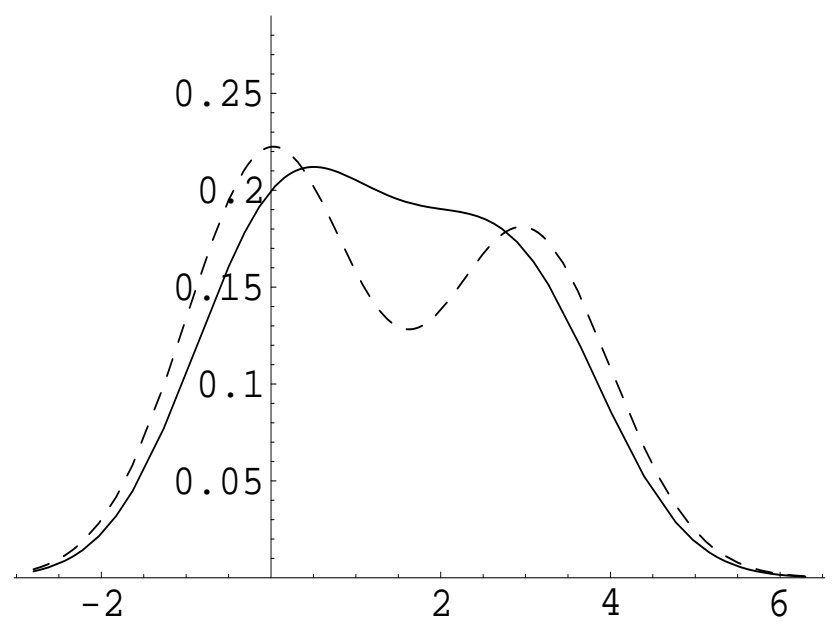

Figure 1: Constructive interference of two Gaussian strategies which are the effect of the tactic $\mathcal{D}_{3}(0.9)$. The dashed line corresponds to the tactic $\mathcal{D}_{3}( \pm 0.9 \mathrm{i})$.

In contrast is the case with $\arg (z)=\pi$. It is represented in Figure 2. Now, after the change of the phase of the $z$ parameter only, the bell like curves which have their origin in the superposition of the Gaussian curves are fully separated. The interference is also the cause of their mutual repulsion. The smaller the gap between the Gaussian components the stronger the influence of the interference is. Figure 3 represents the case with the Gaussian curves of similar height $(z=-\sqrt{0.9})$ which are overlapping in practice $(\Delta=0.2)$. Yet, the destructive interference enhances these features that discriminate between the curves.

The integral curves of the distribution of the probability densities given in Figure 3 (hence the cumulative probability, but with the inverted domain) are represented in Figure 4. In agreement with the previously given market interpretation of the quantum theory [9]-[11] they describe the influence of the quantum (interference) effects onto the shape of the demand curve calculated for the tactic $\mathcal{D}_{0.2}(-\sqrt{0.9})$. 


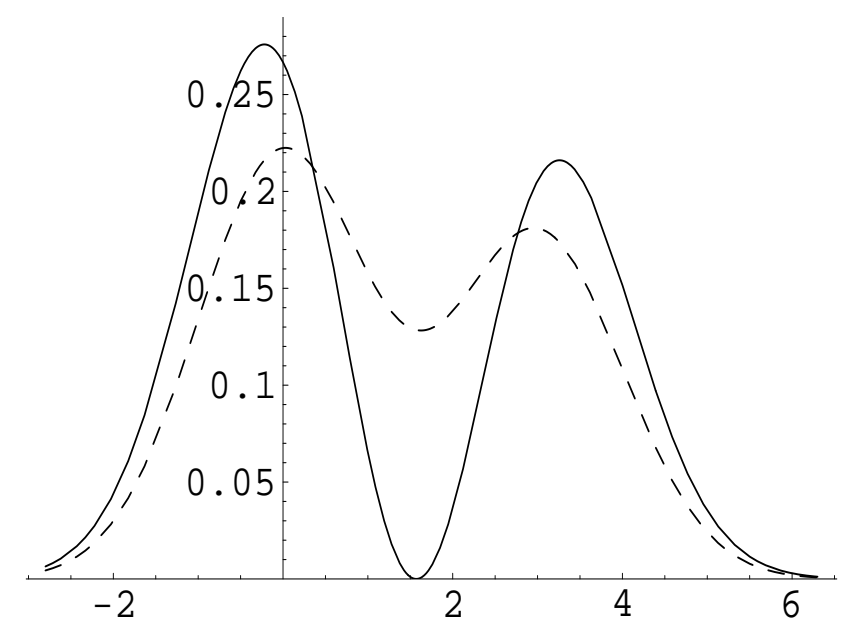

Figure 2: Destructive interference of two Gaussian strategies for the tactic $\mathcal{D}_{3}(-0.9)$. The dashed line corresponds to the tactic $\mathcal{D}_{3}( \pm 0.9 \mathrm{i})$.

\section{Fourier transform of the tactics $\mathcal{D}_{\Delta}(z)$}

We have analyzed the demand following from the strategy which in the demand representation is a superposition of the Gaussian strategies. The description of the strategies in terms of squared modules of the probability amplitudes (as it is in the classical picture) and not the amplitudes themselves is incomplete. Hence it is interesting to complete it by the description of the very strategy in the relation to its supply aspect. The quantum description of the market presented in this paper requires to perceive a player as a subject of a market, who according to the demand representation $\langle q \mid \psi\rangle$ of his strategy $|\psi\rangle$ acquires a specific commodity and consuming it (processing or leaving unchanged) gains a profit described by the operator $\mathcal{Q}+\mathcal{P}$. It is this observable, and not $\mathcal{Q}$ alone, which does not depend on the choice of the monetary unit and measures the price of the commodity in the transaction. The density of the probability of the supply random variable $\mathfrak{p}$ determines the square of the module of the supply representation $\langle p \mid \psi\rangle$ of the same strategy $|\psi\rangle$ according to which the goods has been purchased!

The strategy $|\psi\rangle$, represented by the function $\langle q \mid \psi\rangle$ or its transform $\langle p \mid \psi\rangle$, expresses therefore the full relation of the player with the market. The independent treatment of supply and demand aspects of behaviours appearing 


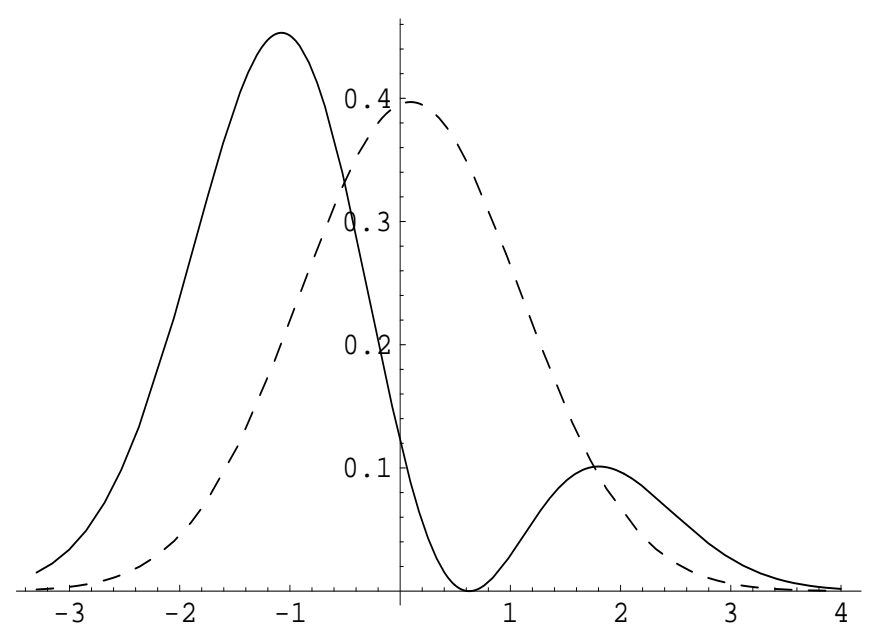

Figure 3: The destructive interference "pushing aside" two of the overlapping Gaussian strategies having similar height - the tactic $\mathcal{D}_{0.2}(-\sqrt{0.9})$. The dashed line corresponds to the tactic $\mathcal{D}_{0.2}( \pm \sqrt{0.9} \mathrm{i})$.

on the market is not in agreement with the quantum description. As far as the interference phenomenon is discussed, the tactics $\mathcal{D}_{\Delta}(z)$ do not commute with the Fourier transformation hence it is not indifferent in which representation, the supply or demand one, the player performs the superposition of the strategies. The supply probability density for the strategies which are the demand superposition of the Gaussian strategies for four different values of their relative phase, that is of the $\operatorname{angle} \arg (z)$, are illustrated in Figures 5 and 6 .

In the case of a player acting as a commercial agent, the derivative of the cumulative probability could be interpreted as the intensity of the sale signal which is sent by the player to the market when he is going to sell a goods purchased beforehand ${ }^{2}$.

We notice that manipulating with the phase allows for a wide choice of the trader's behaviour which characterizes the supply aspect of the tactics $\mathcal{D}_{\Delta}(z)$.

The tactics with $\arg (z)=0$ has the clearly localized sale signal (Figure 5), in the opposition to two equivalent maxima of the sale tactic with $\arg (z)=\pi$.

\footnotetext{
${ }^{2}$ Market games differ a little depending on whether the player announces the will of buying or accepts the price of goods.
} 


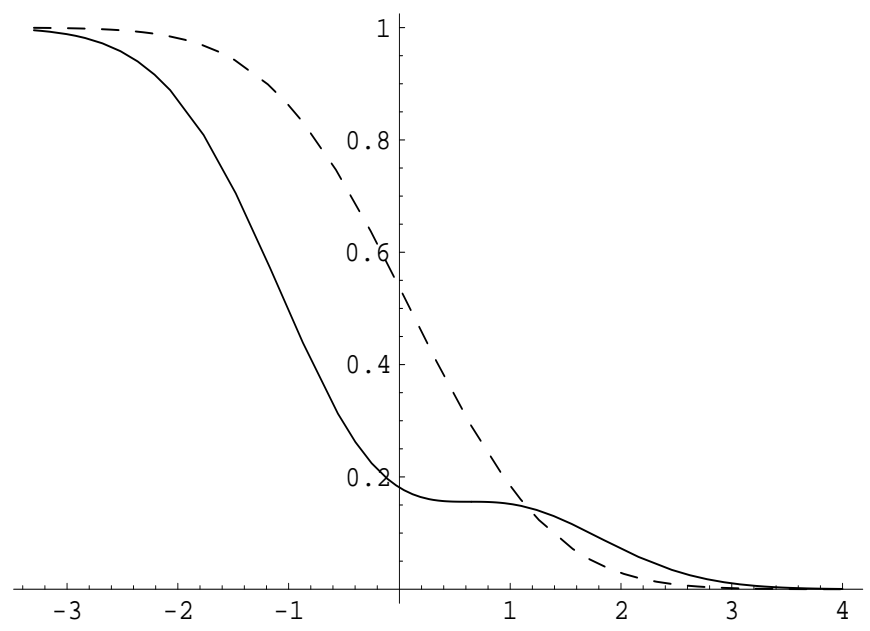

Figure 4: The change of the demand curve caused by the quantum interference effect presented in Figure 3.

For $\arg (z)=\frac{3}{2} \pi$ (Figure 5) we have that with the falling of the price ${ }^{3}$, the buy signal appears at first cautiously, but after a farther decline, it reappears for the second time as a stronger one.

The opposite situation occurs for $\arg (z)=\frac{1}{2} \pi$ (Figure 6). Let us notice that just only the measurement of the random variable $\mathfrak{p}$ allows to distinguish between the strategy $\mathcal{D}_{3}(\mathrm{i})$ and $\mathcal{D}_{3}(-\mathrm{i})$.

\section{The conditional demand}

An interesting thing is the examination of variety of aspects of the strategies which is using the presentation of them in the domain of the phase space $\{p, q\}$ with the help of the Wigner function. For the pure state $|\psi\rangle$ this pseudo-probability density function can be calculated with the following formula

\footnotetext{
${ }^{3}$ The variable $p$ has been chosen so that it diminishes with the increase of the unit price of goods.
} 


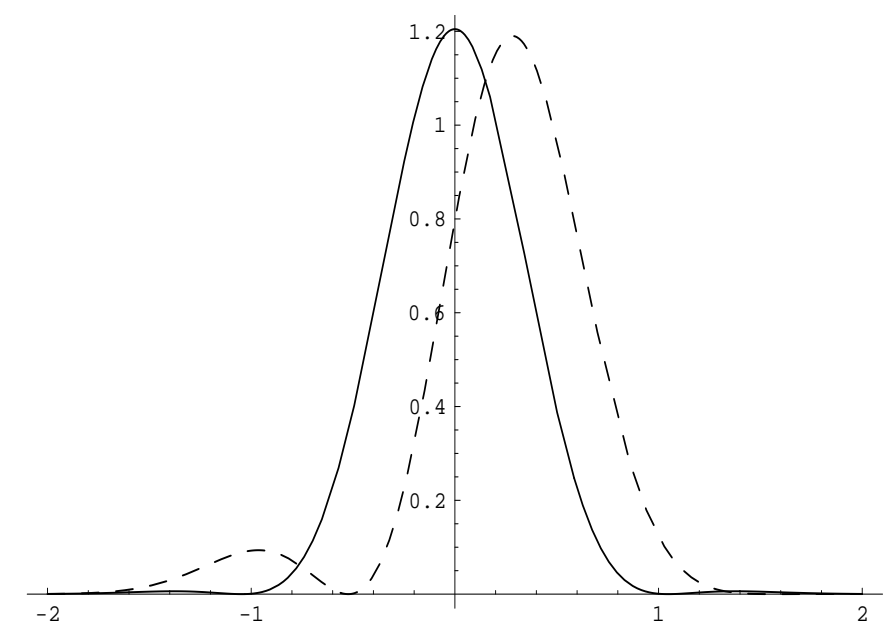

Figure 5: The probability density for the Fourier transforms of the tactics $\mathcal{D}_{3}(1)$ (the continuous line) and $\mathcal{D}_{3}(-\mathrm{i})$ (the dashed line).

$$
\begin{aligned}
W(p, q) & :=h_{E}^{-1} \int_{-\infty}^{\infty} e^{i \hbar_{E}^{-1} p x} \frac{\left\langle q+\frac{x}{2} \mid \psi\right\rangle\left\langle\psi \mid q-\frac{x}{2}\right\rangle}{\langle\psi \mid \psi\rangle} d x \\
& =h_{E}^{-2} \int_{-\infty}^{\infty} e^{i \hbar_{E}^{-1} q x} \frac{\left\langle p+\frac{x}{2} \mid \psi\right\rangle\left\langle\psi \mid p-\frac{x}{2}\right\rangle}{\langle\psi \mid \psi\rangle} d x
\end{aligned}
$$

where $\frac{h_{E}}{2 \pi}$ is the dimensionless economics counterpart of the Planck constant. Figure 7 presents the behaviour of the player who is operating with the market tactic $\mathcal{D}_{0.2}(-\sqrt{0.9})$.

Note that the asymmetric crater-like hollow in the diagram has the minimum bellow zero, the fact which qualitatively distinguishes the Wigner function from the supply and demand distributions for models formulated in the realm of the classical probability theory in which the measure of the probability has to be nonnegative. The intersection of the surface of the diagram with the surface given by $p=$ constant depicts the conditional probability density which is the measure of the probability for the withdrawal price of the player in the situations when this price is constant during the act of selling. The cross sections for the negative values of the Wigner function are characteristic for the situation of the giffen-strategy. The suitable integrals for these curves represent fully rational situations for which the demand (or 


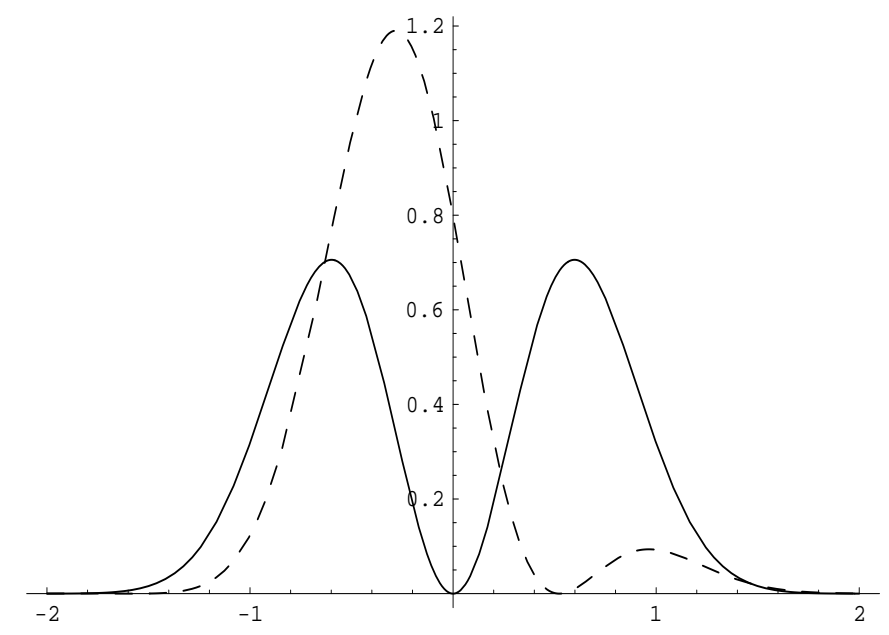

Figure 6: The probability density for the Fourier transforms of the tactics $\mathcal{D}_{3}(-1)$ (the continuous line) i $\mathcal{D}_{3}(\mathrm{i})$ (the dashed line).

supply) cease to be a monotonous function. The example of such a reaction of the player (it might be the rest of the world) is illustrated in Figure 8. We observe here the lack of the property of the monotonicity for the demand (or supply) curves (Giffen paradox). In this context it is worthy to raise the question whether the legendary captain Giffen, after observing a market anomaly which is contradictory to the law of demand, has recorded the surprising (although having an explanation) demand that decreases after the fall of the price, or simply noticed the destructive interference which had been the effect of a careful demand transformation characteristic for a intelligent (hence acting rationally) but poor consumer. The authors incline towards the second answer. It has the superiority because of its ability to be falsified [13] which is a consequence of the precision qualitative predictions for this phenomenon made by the quantum theory. The case of the non-monotonous conditional demand for interfering Gaussian strategies has been characterized before. We recall that this characteristic is the proper one for all non-Gaussian strategies. Therefore it seems important to look after the conditions of the market game under which the strategies described by the normal distributions do not lead to the maximalization of value of the intensity of the gain. They might explain the circumstances in which we met the giffen-strategies. 


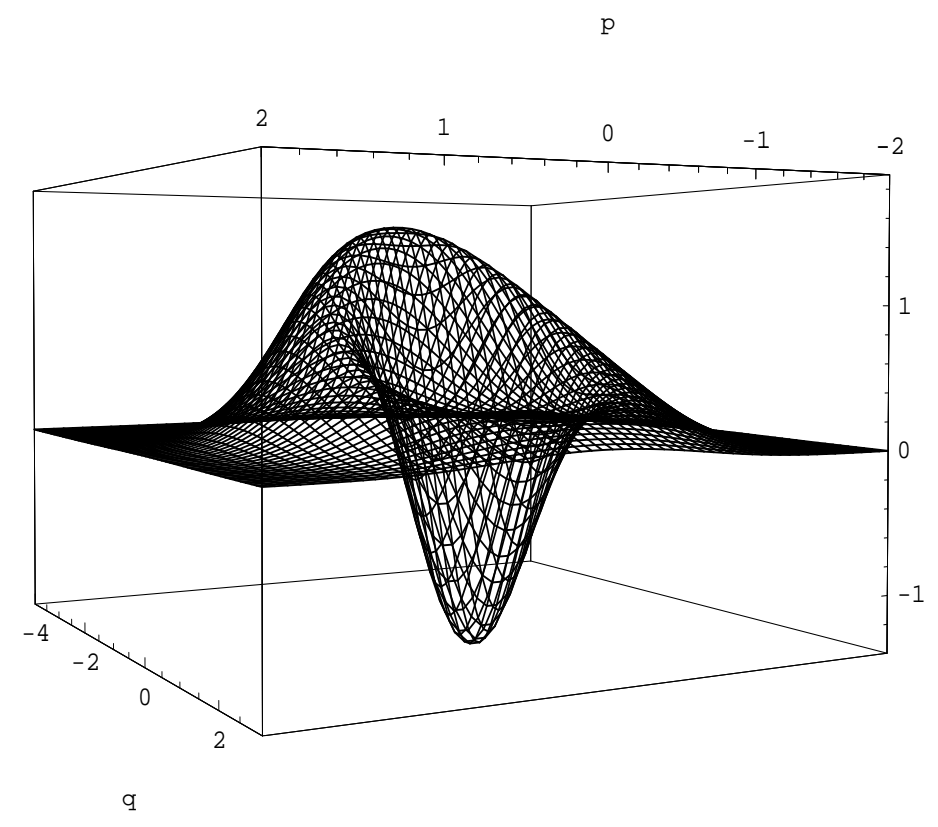

Figure 7: Plot of the Wigner function for the tactic $\mathcal{D}_{0.2}(-\sqrt{0.9})$.

The careful reader has certainly noticed other, besides the non-monotonicity, characteristic of the integral curves for some cross sections of the diagrams of the Wigner function. They have global maxima for definite values of the parameter $q=q_{\max }$ (eg, for the surface in Figure 7 with the opposite orientation of the axis $p$ ). It means that there exists a nonzero price of the commodity $\mathrm{e}^{\frac{q_{\max }-q_{0}}{\sigma}}$ under which the player irreversibly losses his interest in its buying. For example in the capricious world of the fashion business such a kind of market behaviours are frequently noticed; they are also known by the owners of the rubbish auction. The example of a conditional demand curve is presented in Figure 9. When, under the condition of unchanging tactic player's estimation of the usefulness of the goods has diminished properly, and afterwards the value of the parameter $p$ has been changed, then the new cross section shall be free from this anomaly.

Both of the just discussed phenomena of the departures from the law of demand and supply (and also others paradoxical properties) disappear if only the cross sections are integrated over $p$. The thus obtained joint cumulative probability of demand, being the integral of the square of the module of the 


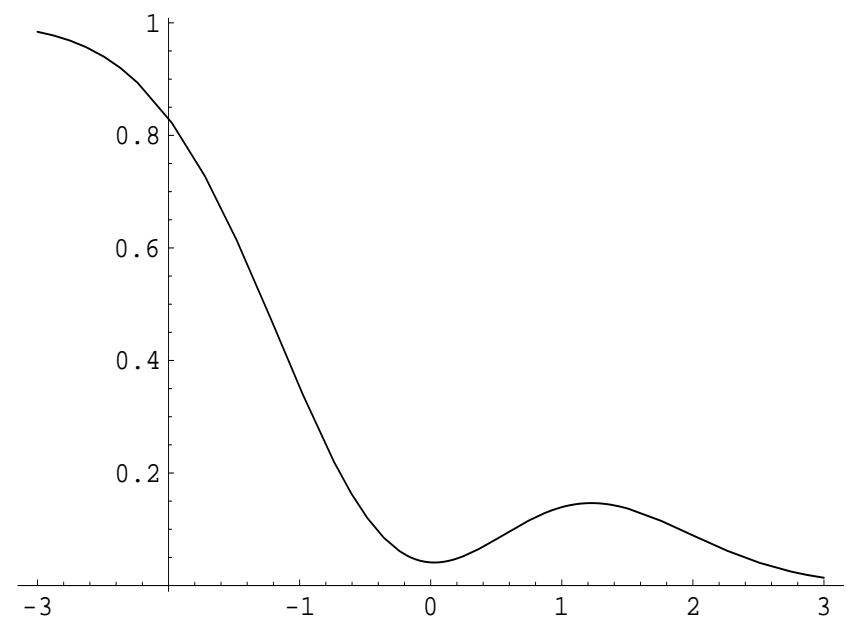

Figure 8: The non-monotonous conditional demand for the tactic $\mathcal{D}_{0.2}(-\sqrt{0.9})$ (The integral curve for the intersection of the surface from Figure 7 with the plain $p=0.4\left[\frac{\hbar_{E}}{\sigma}\right]$ ).

function $\langle q \mid \psi\rangle$, fulfills the orthodox form of the law.

The above considerations have proved that the superposition of the elementary quantum strategies has peculiarities which are well known from the observations of markets. The modeling of these behaviours in the realm of the traditional probability theory would be impossible for the same reason that made the physicists to reject the classical picture of the world. In this context it seems obvious that eg the designers of the software, which automatically invests on capital markets, sooner or later will be confronted with the need of using models of quantum market games. Hence it is worthy to make a few comments on the unique efficacy which is characteristic for the quantum tactics.

\section{Self-consistent nonlinear tactics}

In Ref. [12] the nonlinear tactics $\mathcal{D}_{E(\mathfrak{q})}$ operating on the Dirac strategy which could be perceived as a limit of Gaussian strategies for $\sigma \rightarrow 0$ were analyzed. Due to the unique property which is connected with the fixed point theorem [12] it appears to be the method of the maximization of the gain 


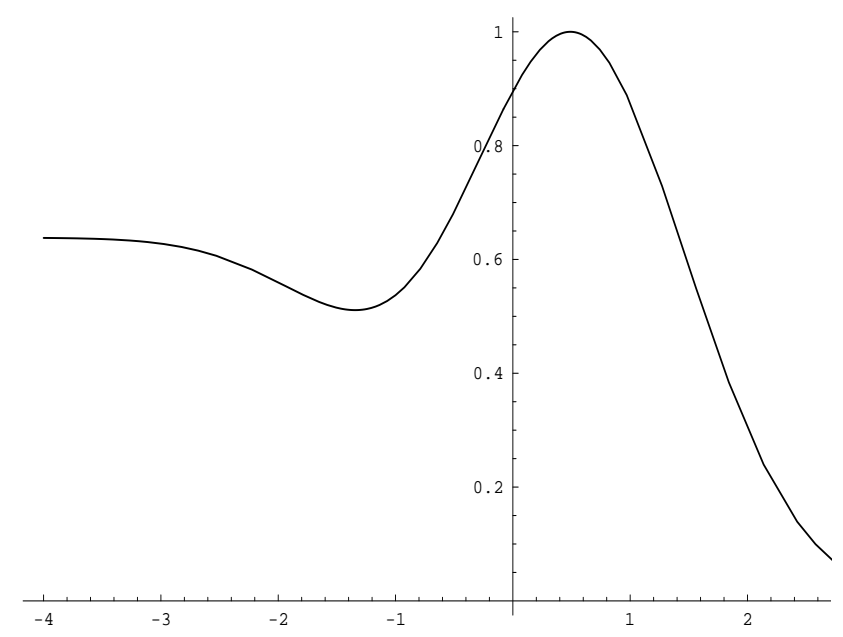

Figure 9: The non-monotonous conditional demand with the maximum for the nonzero value of the price. The tactic $\mathcal{D}_{0.2}\left(-0.9^{-\frac{1}{2}}\right)$ (the integral curve for the cross section of the surface from Figure 7 with the plane $\left.p=-0.2\left[\frac{\hbar_{E}}{\sigma}\right]\right)$.

in the market game, in which the rest of the world, being in a polarization compelling to the exhibition of the offers of prices, accepts the passive tactic $\mathcal{I}$ on its own Gaussian tactic. The tactic $\mathcal{D}_{E(\mathfrak{q})}$ requires the measurement of the mean $E(\mathfrak{q})$ hence it can not be used in a single game. Yet, in the sequence of identical games, when a partner behaves in a passive manner, we are able, in view of the past experiences, to approximate the parameter $E(\mathfrak{q})$ so that in the following one to shift our withdrawal price to this value. The tactic $\mathcal{D}_{E(\mathfrak{q})}$ understood in this way is then the unitary one, and being the quantum operation guarantees to us the conditions required for being confidential. The just mentioned fixed point theorem assumes that measure of the probability given by the opponent's tactic is positive definite. It is possible that if an obligatory passive opponent (e.g. the rest of the world is not able to react to the inconvenient behaviour of only one small participant of the market) chooses the giffen-strategy then the tactic $\mathcal{D}_{E(\mathfrak{q})}$ losses the quality of the most beneficial one. The infancy of quantum computers do not hinder us from simulating the scenarios of quantum games on the traditional computers. In such situations the use of the simplest quantum tactics which are similar to the ones presented here should bring the completely new qualitative effects. These tactics are more rich than the hardly similar to the 
alive human market behaviour present algorithms for automatic investment. Even today the quantum strategies might be used on capital markets if only funds are raised to be managed in the quantum manner. Also nothing is opposing to the possibility that new markets appear, on which all parties of transactions might be using quantum tactics. The physical quantum models offer the solutions which are extremely stable in comparison with their classical counterparts [14]. So, perhaps the quantum markets would protect the future society from negative consequences of the deep oscillations of the prices which are characteristic for nowadays economy.

For the needs of the time, when the principles of the functioning of the market allow to analyze behaviors of partners of transactions, the game, which is played with an opponent whose tactic is reacting to our changes of the strategy, should be examined. Such games posses the thermodynamic analogies and allows to describe processes of reaching the market equilibrium. Because of the extension of the subject, the analysis of the competitive market tactics exceeds the limits of this paper which sketches only the theme of the quantum market games.

\section{Conclusions}

When the player has at his disposal two pure strategies $\left|\psi_{0}\right\rangle$ and $\left|\psi_{1}\right\rangle$ then the set of the classical strategies in which (going from $\left|\psi_{0}\right\rangle$ to $\left|\psi_{1}\right\rangle$ ) he can make changes in a continuous way, is limited to the one dimensional set of the mixed strategies spanned over the points $\left|\psi_{0}\right\rangle$ and $\left|\psi_{1}\right\rangle$. In comparison to this, the quantum theory offers him extremely reach possibilities. He or she faces a choice of a one from many optional continuous curves for many of the paths connecting the poles of the sphere $S_{2}$. Therefore such a "quantum player" puts the qualitatively new challenges to an opponent. The opponent who has at his disposal the knowledge based on the classical game theory only, often is doomed from the start.

\section{References}

[1] J. Eisert, M. Wilkens, and M. Lewenstein, Phys. Rev. Lett. 83 (1999) 3077.

[2] D. Meyer, Phys. Rev. Lett. 82, (1999) 1052. 
[3] Marinatto, L. \& Weber, T., A quantum approach to static games of complete information, Phys. Lett. A 272 (2000) 291-293.

[4] Iqbal, A. \& Toor, A.H., Evolutionary stable strategies in quantum games, Phys. Lett. A 280 (2001) 249-256.

[5] Iqbal, A. \& Toor, A.H., Quantum Mechanics gives Stability to a Nash Equilibrium, Phys. Rev. A in press.

[6] Iqbal, A. \& Toor, A.H., Entaglment and Dynamic Stability of Nash Equilibria in a Symmetric Game, Phys. Lett. A, 286 (2001) 245-250.

[7] Iqbal, A. \& Toor, A.H., Backwards-induction Outcome in a Quantum Game; quant-ph/011090.

[8] Piotrowski, E.W. \& Sładkowski, J. Quantum-like approach to financial risk: quantum anthropic principle, Acta Phys. Pol. B32 (2001) 38733879; quant-ph/0110046.

[9] Piotrowski, E.W. \& Sładkowski, J. Quantum bargaining games Physica A 308 (2002) 391; quant-ph/0107140.

[10] Piotrowski, E.W. \& Sładkowski, J. Quantum market games, Physica A in press; quant-ph/0104006.

[11] Piotrowski, E.W. \& Sładkowski, J. Quantum English Auctions, submitted to Physica A; quant-ph/0108017.

[12] E. W. Piotrowski and J. Sładkowski, submitted to Ann. of Statistics, cond-mat/0102174.

[13] Popper, K. R., The Logic of Scientific Discovery, HARPER, New York (1968).

[14] Deutsch, D., The Fabric of Reality, PENGUIN, London (1998). 